\begin{tabular}{|c|l|}
\hline Title & Stripe formation in an immiscible polymer blend under electric and shear-flow fields \\
\hline Author(s) & Na, Yang-Ho; Shibuya, Tetsunori; Ujiie, Seiji; Nagaya, Tomoyuki; Orihara, Hiroshi \\
\hline Citation & $\begin{array}{l}\text { Physical Review E, 77(4), 041405-1-041405-4 } \\
\text { https://doi.org/10.1103/PhysRevE.77.041405 }\end{array}$ \\
\hline Issue Date & 2008-04 \\
\hline Doc URL & http://hdl.handle.net/2115/50759 \\
\hline Rights & ○ 2008 A merican Physical Society \\
\hline Type & article \\
\hline File Information & Phys. Rev. E 77, 041405.pdf \\
\hline
\end{tabular}

Instructions for use 


\title{
Stripe formation in an immiscible polymer blend under electric and shear-flow fields
}

\author{
Yang-Ho Na, ${ }^{1}$ Tetsunori Shibuya, ${ }^{2}$ Seiji Ujiie, ${ }^{3}$ Tomoyuki Nagaya, ${ }^{4}$ and Hiroshi Orihara ${ }^{1, *}$ \\ ${ }^{1}$ Department of Applied Physics, Graduate School of Engineering, Hokkaido University, Sapporo 060-8628, Japan \\ ${ }^{2}$ Department of Applied Physics, Graduate School of Engineering, Nagoya University, Nagoya 464-8603, Japan \\ ${ }^{3}$ Department of Applied Chemistry, Faculty of Engineering, Oita University, Oita 870-1192, Japan \\ ${ }^{4}$ Department of Electrical and Electronic Engineering, Faculty of Engineering, Oita University, Oita 870-1192, Japan
}

(Received 11 November 2007; published 18 April 2008)

\begin{abstract}
We found a stripe formation in an emulsion of a liquid crystalline polymer (LCP) and a machine oil (OIL) in electric and shear fields. Through the simultaneous measurement with a confocal scanning laser microscope and a rheometer, it was clearly shown that the formation of stripes, which are periodically arrayed, leads to the increase of the shear stress. The droplets, which are one component of the emulsion, start to be connected at low electric fields and then change into the stripes with the increase of electric field. Finally, a threedimensional network is formed at high electric fields. The period and fluctuation of the stripe structure were also investigated in detail.
\end{abstract}

DOI: 10.1103/PhysRevE.77.041405

PACS number(s): 82.70.Kj, 83.10.Tv, 83.80.Tc

\section{INTRODUCTION}

Typical electrorheological (ER) fluids are suspensions of highly polarizable particles in an insulating liquid [1,2]. Their rheological properties are dramatically changed upon the application of an electric field. The electric field of the order of $1 \mathrm{kV} / \mathrm{mm}$ can increase the apparent viscosity by several orders of magnitude. As this drastic change of rheology is electrically controlled, these ER fluids are very important for commercial applications. This rheological behavior is related to the microstructure change of ER fluids. It is worth noting that the applied fields such as electric and shear fields induce pattern formation. Without shear flow, the electric field makes the formation of particle columns oriented in the direction of the field $[1,3]$, whereas, with shear flow, the field makes the formation of particle stripes oriented in the flow direction [4-9]. Such a stripe or layer formation in ER suspensions was reported in some experiments under simple shear flow [4-6]. Numerical simulation based on a dipole model [7], a two-fluid continuum model [8], and an idealized electrostatic polarization model [9] also showed the pattern formation when ER fluids were subjected simultaneously to an electric field and shear flow. Volkova et al. [5] observed an abrupt jump of stress and the onset of a layered stripe pattern above a critical shear rate.

Immiscible liquid blends also exhibit a large ER effect [10-14]. Under the application of an electric field, remarkable changes of viscosity were reported when the blend has a mismatch of electrical properties such as permittivity and conductivity between the two fluids. Such a blend usually consists of two liquids having different viscosities; one with high viscosity is dispersed as droplets in the other with low viscosity. In the absence of an electric field, the apparent viscosity is low because the droplets can flow easily sliding over each other in the matrix of the low-viscosity fluid. When subjected to an electric field, the droplets of the high-

\footnotetext{
*Author to whom correspondence should be addressed; orihara@eng.hokudai.ac.jp
}

viscosity fluid become elongated along the field due to the electrostriction exerted on the interfaces, and join together to form bridges in the direction of the electric field. This may result in an apparent increase in viscosity.

However, the direct observation was difficult under shear flow. Recently, we have succeeded in investigating the morphology in immiscible blend ER fluids [15-19]. Especially, detailed observations have been performed by using a specially designed shearing apparatus and a confocal scanning laser microscope $[18,19]$. The polymer blends showed morphological change from a droplet-dispersed structure to a network structure under electric and shear fields. The differences of viscosity, permittivity, and conductivity between the two fluids are crucial parameters in the morphological changes. The structure change is mainly associated with the coalescence of the high-viscosity phase, which cannot occur in suspension of solid particles.

In this paper, we report a stripe formation in an immiscible blend when it is subjected to electric and shear fields. The effect of external field on the stripe structure and its fluctuation is studied.

\section{EXPERIMENT}

In this study, we used a liquid crystalline polymer (LCP) and a machine oil (OIL). The LCP was supplied by Asahi Chemical Industry (Japan) and the OIL (NCL 320) was purchased from Taniguchi Sekiyu Company (Japan). All the experiments were done at room temperature, where the LCP is in an isotropic phase, indicating that the ER effect in our blend is not directly related to the orientational change of the mesogens. The viscosities and permittivities of LCP and OIL are, respectively, $60 \mathrm{~Pa} \mathrm{~s}, 0.32 \mathrm{~Pa} \mathrm{~s}, 10$, and 2.3.

We made a blend with LCP and OIL in a weight ratio of 1:4. To observe the structure clearly and distinguish LCP from OIL, a small amount of fluorescent dye, $\mathrm{N}, \mathrm{N}^{\prime}$ - dimethyl- $\mathrm{N}$ - (iodoacetyl) - $\mathrm{N}^{\prime}$ - (7 - nitrobenz-2 - oxa- 1 , 3-diazol-4-yl)ethylenediamine (IANBD amide, Molecular Probes) was doped to the LCP before it was mixed with the OIL. Previously, we confirmed that the dye did not dissolve 

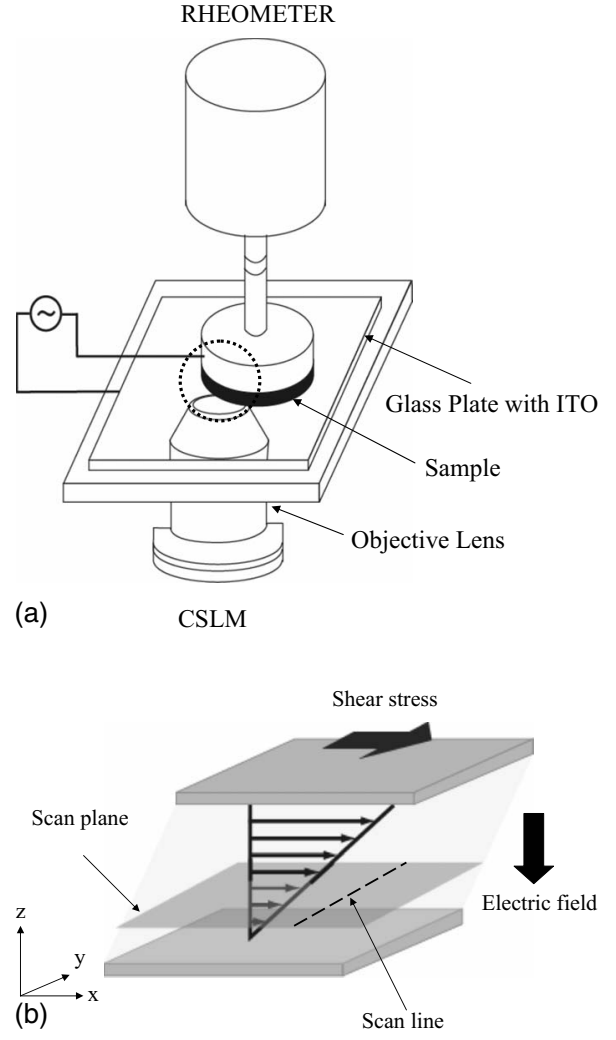

FIG. 1. (a) Schematic illustration of the system combining a confocal scanning laser microscope and a rheometer; (b) the flow behavior and scanning area in it.

in the OIL. The wavelength of excitation was $488 \mathrm{~nm}$ and a low-pass filter of $520 \mathrm{~nm}$ for the detection of light was used. This mixture was prepared by dissolving them in tetrahydrofuran followed by evaporation of the solvent under vacuum. The blend was sandwiched between a bottom glass plate with an indium-tin-oxide (ITO)-coated electrode and the rotating metal disk of a rheometer (M10 and RS20, Haake). A schematic picture of the system is shown in Fig. 1(a). The diameter of the rotating disk and the gap between the two parallel plates were 20 and $0.2 \mathrm{~mm}$, respectively. Since we used a parallel-plate rotational viscometer and so the shear rate depends on its position, the shear rate was defined as the one at the periphery of the upper disk. The shear stress at the edge of the top plate was calculated from the torque by assuming that the fluid is Newtonian, though it is a crude approximation in our fluids. The structure of the blend was observed through the ITO glass plate by a confocal scanning laser microscope (CSLM, Fluoview FV300, Olympus). Figure 1(b) depicts the scanning area and the flow behavior inside the system. We adopted two types of scans. A twodimensional scan was made in the $x-y$ plane, where $x$ is along the flow direction and $y$ is parallel to the vorticity. The maximum scan rate is about 4 frames/s for 512 $\times 512$ pixels. The scan rate was so slow that the obtained images were distorted by the flow. In order to overcome this difficulty, we made a one-dimensional scan along a line perpendicular to the flow direction ( $y$ direction). It took only $2.02 \mathrm{~ms}$ to scan one line (512 pixels) once. We scanned the line repeatedly. Since the blend moved by the flow perpen-

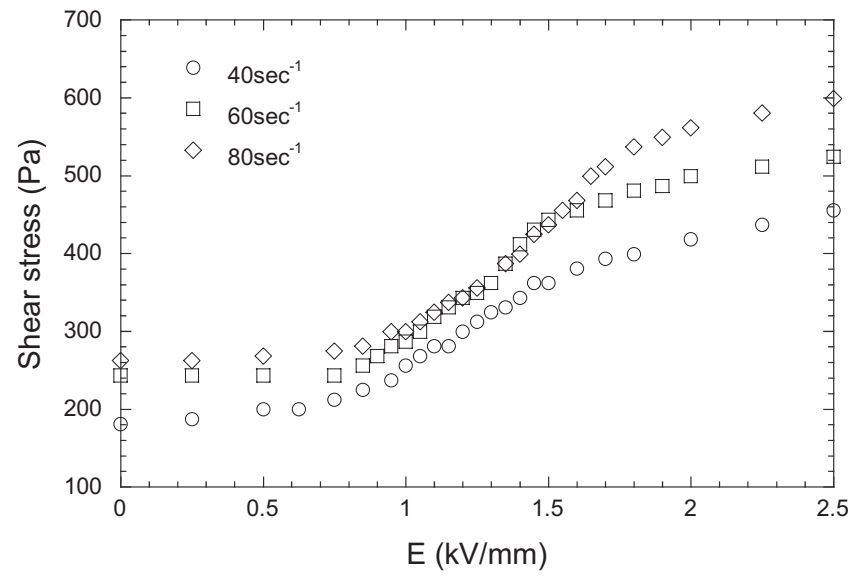

FIG. 2. Electric field dependences of shear stress at different shear rates.

dicular to the line, we could construct a two-dimensional image.

Electric fields were applied to the blend by a synthesizer (model 1940, NF Electric Instruments) and a high voltage amplifier (model 609C-6, Trek). An ac electric field of $10 \mathrm{~Hz}$ was applied to avoid the migration of ionized LCP droplets. Before the measurements, we applied a shear of $100 \mathrm{~s}^{-1}$ and an electric field of $2 \mathrm{kV} / \mathrm{mm}$ for $2 \mathrm{~min}$ and then continued to apply a shear of $100 \mathrm{~s}^{-1}$ for 2 min under no electric field to make a LCP-droplet dispersed structure.

\section{RESULTS AND DISCUSSION}

Figure 2 shows the electric field dependences of shear stress at different shear rates. The shear stress hardly changes at low electric fields, then gradually increases at the intermediate fields, and then slowly reaches to a saturation value at high fields. In our previous paper [18] it was shown that LCP is dispersed as droplets under no electric field, but with an application of electric fields the droplets are elongated and coalesce to form a network structure, resulting in the viscosity increase. In the present experiment, we investigated in detail the height $(z)$ dependence of the structure under electric fields.

Figure 3 shows a $y-z$ cross section constructed from a series of $x-y$ scan images obtained at a shear rate of $40 \mathrm{~s}^{-1}$ and an electric field of $2.0 \mathrm{kV} / \mathrm{mm}$. It is seen that the structure changes depending on the height and three-dimensional layered stripes are formed from the glass surface up to about $15 \mu \mathrm{m}$. It seems that these stripes become unstable far from

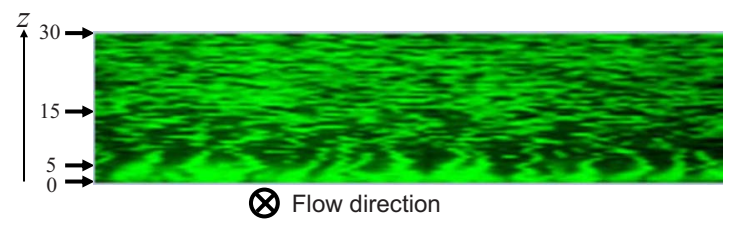

FIG. 3. (Color online) View of the $y-z$ cross section constructed from a series of $x-y$ scan images at a shear rate of $40 \mathrm{~s}^{-1}$ and an electric field of $2.0 \mathrm{kV} / \mathrm{mm}$. 


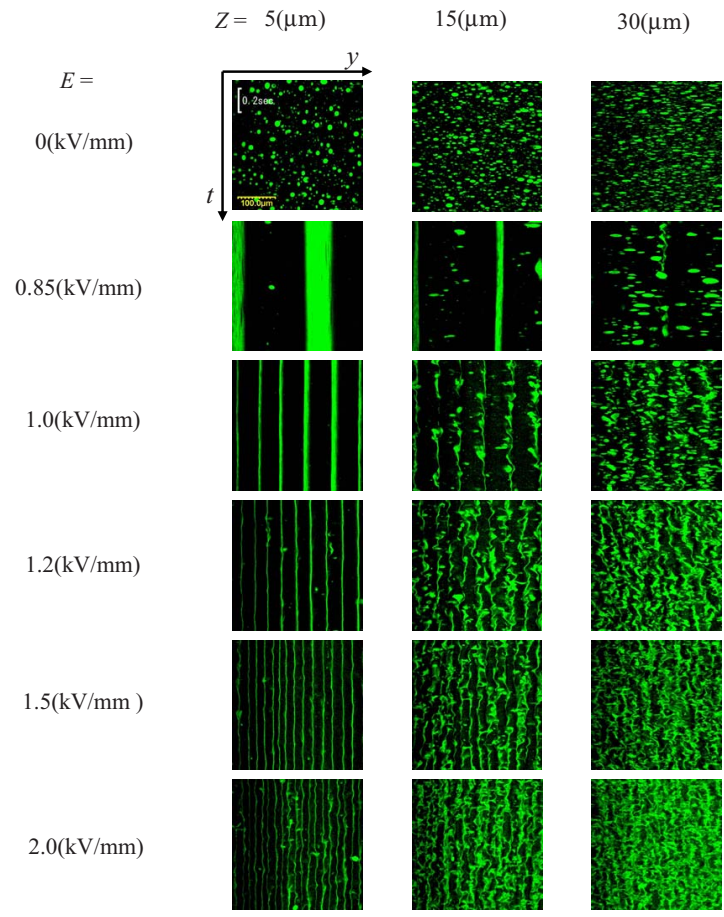

FIG. 4. (Color online) Views of the one-dimensional scans at several heights and electric fields at a shear rate of $40 \mathrm{~s}^{-1}$.

the glass surface, and then a three-dimensional network is made above $z=15 \mu \mathrm{m}$. As has been pointed out [18], the network is not static but dynamic, and so the linkages are at some time and at some place formed and destroyed continually.

The formation mechanism of stripes in our emulsion system may be similar to that in the suspension system. Pfeil et al. have shown that the formation of particulate stripes in electric and shear flow fields is related to the column formation in an electric field without shear flow [8]. The columns are formed by the fluctuations of particle concentration parallel to the applied field, which are unstable, while the perpendicular fluctuations are stable. In shear flow, the unstable parallel fluctuations will be rotated toward the flow direction to become the stable perpendicular ones, resulting in a uniform concentration in the plane of shear. The above scenario may be applicable to our emulsion system, because the column (bridge) formation takes place also in our system without shear flow.

The image in Fig. 3 was not so clear, because the twodimensional scan cannot follow the structural change. We tried a one-dimensional scan to obtain a higher time resolution. Figure 4 shows the results of one-dimensional scans at several heights and electric fields at a shear rate of $40 \mathrm{~s}^{-1}$, where the ordinate is the time but may be regarded as $x$ axis if the blend moves without changing mutual positions and multiplied by the flow velocity at each height. Without electric field, droplets are seen. It should be noted that the droplets are more deformed at higher positions, where the flow velocity becomes larger, because of the contraction along the $t$ axis. When an electric field is applied, a striped pattern appears at $z=5$ and $15 \mu \mathrm{m}$. As the electric field is raised, the period of the stripe becomes shorter, but it is almost the same

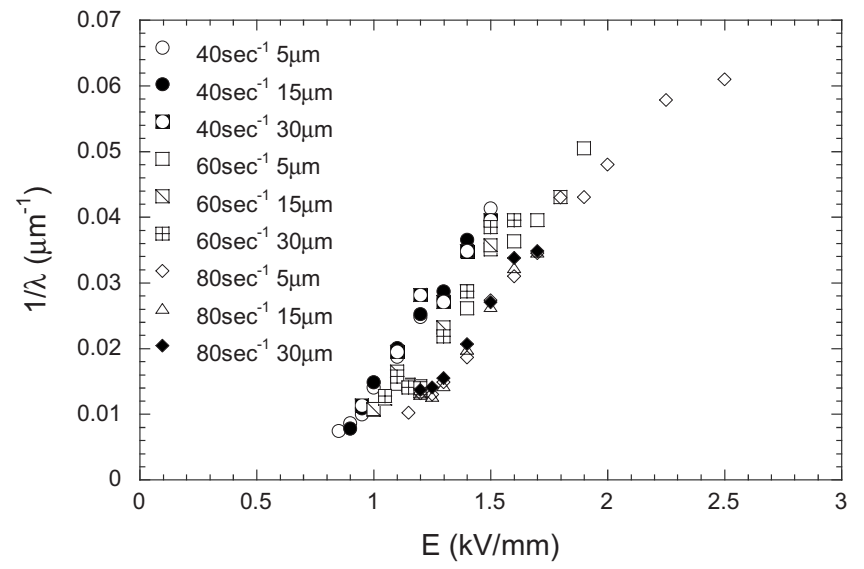

FIG. 5. Dependences of the reciprocal of period, $1 / \lambda$, on electric field at different shear rates and heights.

at $z=5$ and $15 \mu \mathrm{m}$. At each electric field, the lines become blurred as the height increases. At $z=30 \mu \mathrm{m}$, almost no stripe is seen and the droplet-dispersed structure changes into a connected structure or a network as the electric field is increased. On the other hand, at $15 \mu \mathrm{m}$ the lines begin to fluctuate with increasing the electric field, and around $1.2 \mathrm{kV} / \mathrm{mm}$ they begin to be connected in the direction perpendicular to the flow. The tendency of the structural changes at 60 and $80 \mathrm{~s}^{-1}$ was similar to that at $40 \mathrm{~s}^{-1}$, but the electric field to bring about the structural change shifts to higher ones.

As has been previously shown, the stripe pattern changes in the period and the linewidth. To investigate the dependences of them on the electric field, the shear rate, and the height, we made image analyses [National Institutes of Health $(\mathrm{NIH})$ image]. Let $I(y, t)$ be the intensity at the position $y$ and time $t$. First, we calculated the average of $I(y, t)$ over the time, $\bar{I}(y)=\langle I(y, t)\rangle_{t}$. The period $\lambda$ was successfully obtained from the power spectrum of $\bar{I}(y)$. However, the linewidth (stripe) including the blur was difficult to obtain from it. We defined the linewidth $w$ in the following equation:

$$
\bar{I}(y)=\sum_{n=-\infty}^{\infty} a \exp \left[-(y-n \lambda)^{2} /\left(2 w^{2}\right)\right]
$$

where $a$ is a constant, and obtained a ratio $w / \lambda$ as follows. Note that $\bar{I}(y)$ is almost constant for $w / \lambda>0.5$. It is easily shown that the Fourier coefficient of $\bar{I}(y)$, by substituting Eq. (1),

$$
\begin{aligned}
c_{m} & =\frac{1}{\lambda} \int_{-\lambda / 2}^{\lambda / 2} \bar{I}(y) \exp \left(i \frac{2 \pi m}{\lambda} y\right) d y \\
& =a \sum_{n=-\infty}^{\infty} \int_{-1 / 2}^{1 / 2} \exp \left[-(y-n)^{2} / 2\left(\frac{w}{\lambda}\right)^{2}\right] \exp (i 2 \pi m y) d y,
\end{aligned}
$$

has a functional form as $a f(w / \lambda)$. Therefore, $\lambda / w$ can be determined from, for example, $c_{0} /\left|c_{1}\right|$. We calculated $c_{0} /\left|c_{1}\right|$ from an image using $\lambda$ obtained from the power spectrum, 


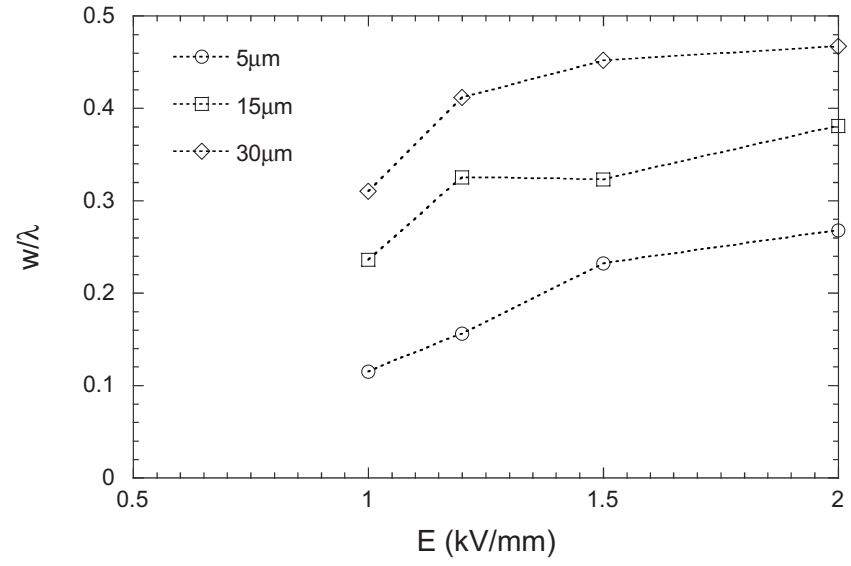

FIG. 6. Dependences of $w / \lambda$ on electric field, $E$, at different heights at a shear rate of $40 \mathrm{~s}^{-1}$.

and then obtained $\lambda / w$ from the numerical curve of $\lambda / w$ vs $c_{0} /\left|c_{1}\right|$ calculated from Eq. (2).

Figure 5 shows the electric field dependences of the reciprocal of period, $1 / \lambda$, at different shear rates and heights. It is found that $1 / \lambda$ linearly increases with $E$ and it is almost independent of the height. Moreover, there are threshold electric fields, where the striped pattern begins to appear. The thresholds are $0.73,0.80$, and $0.90 \mathrm{kV} / \mathrm{mm}$ at 40,60 , and $80 \mathrm{~s}^{-1}$, respectively. Figure 6 shows the dependency of $w / \lambda$ on $E$ at the shear rate of $40 \mathrm{~s}^{-1}$. The $w / \lambda$ increases as the electric field and the height increase. It is clearly seen that $w / \lambda$ depends on the height and the networks are formed above $w / \lambda \simeq 0.4$. The results of $w / \lambda$ at 60 and $80 \mathrm{~s}^{-1}$ were similar to that at $40 \mathrm{~s}^{-1}$, though they were not shown here.

These results of Fig. 6 may explain the height dependence of the pattern. When the electric field is applied, LCP droplets near the glass surface adhere to it. The affinity of LCP for the ITO on the glass plate may be good and therefore they remain on it to form stripes along the flow. The stripes may be stable because they are fixed on the glass plate.
While at high positions, the stripes (layers) may be unstable due to the shear flow and broken into droplets at low electric fields. While at high electric fields, the stripes can be maintained but the fluctuations become large to form a network structure.

The observation with CSLM is limited to $30 \mu \mathrm{m}$ from the glass plane and the entire observation is very difficult. From the symmetrical point of view, we suggest that the structural change near the rotating plate at the top may be similar to that near the bottom glass plate.

\section{SUMMARY}

We investigated structural changes and rheological properties in an immiscible blend in electric and shear flow fields. It turned out that with increasing the electric field the shear stress hardly changes at low electric fields, gradually increases at intermediate fields, and then slowly reaches to a saturation value at high fields. Through the observation with a CSLM, it was clarified that the formation of the stripes which are periodically arrayed near the glass plate leads to the increase of the shear stress in the intermediate field region. In the high field region, the droplets are connected also in the direction perpendicular to the flow to make a threedimensional network. Further studies are now in progress to theoretically elucidate the formation mechanism and the electric field dependence of the stripe pitch.

\section{ACKNOWLEDGMENTS}

The authors would like to thank Dr. A. Inoue of ERtec Co., Ltd. for supplying the LCP. This work was partially supported by Grant-in-Aid for Scientific Research [Priority Area "Soft Matter Physics" (Contract No. 463), C (Contract No. 19540326), and Wakate B (Contract No. 19740255)] from the Ministry of Education, Culture, Sports, Science and Technology of Japan.
[1] W. M. Winslow, J. Appl. Phys. 20, 1137 (1949).

[2] T. C. Halsey, Science 258, 761 (1992).

[3] M. Parthasarathy and D. J. Klingenberg, Mater. Sci. Eng., R. R17, 57 (1996).

[4] S. Henley and F. E. Filisko, J. Rheol. 43, 1323 (1999).

[5] O. Volkova, S. Cutillas, and G. Bossis, Phys. Rev. Lett. 82, 233 (1999).

[6] S. L. Vieira, L. B. Pompeo Neto, and A. C. F. Arruda, J. Rheol. 44, 1139 (2000).

[7] J. M. Sun and R. Tao, Phys. Rev. E 53, 3732 (1996).

[8] K. von Pfeil, M. D. Graham, D. J. Klingenberg, and J. F. Morris, Phys. Rev. Lett. 88, 188301 (2002).

[9] H. G. Sim, K. H. Ahn, and S. J. Lee, J. Rheol. 47, 879 (2000).

[10] K. Tajiri, H. Orihara, Y. Ishibashi, M. Doi, and A. Inoue, J. Rheol. 41, 335 (1997).

[11] A. Inoue, Y. Ide, and H. Oda, J. Appl. Polym. Sci. 64, 1319
(1997).

[12] H. Kimura, K. Aikawa, Y. Masubuchi, J. Takimoto, K. Koyama, and K. Minagawa, Rheol. Acta 37, 54 (1998).

[13] X. Pan and G. McKinley, J. Colloid Interface Sci. 195, 101 (1997).

[14] J. Ha and S. Yang, J. Rheol. 44, 235 (2000).

[15] H. Orihara, Y. Hosoi, K. Tajiri, Y. Ishibashi, M. Doi, and A. Inoue, J. Rheol. 43, 125 (1999).

[16] K. Tajiri, H. Orihara, Y. Ishibashi, M. Doi, and A. Inoue, J. Rheol. 43, 137 (1999).

[17] H. Orihara, Y. Ikeyama, S. Ujiie, and A. Inoue, J. Rheol. 47, 1299 (2003).

[18] H. Orihara, T. Shibuya, T. Nagaya, and S. Ujiie, J. Phys. Soc. Jpn. 75, 063802 (2006).

[19] Y.-H. Na, A. Yoshino, S. Tominaga, H. Orihara, S. Ujiie, and T. Nagaya, Nihon Reoroji Gakkaishi 34, 199 (2006). 